\section{Las doce teorías que fundamentan la combinación y la unificación de la Psicoterapia}

\author{
José Félix Cosme López Salinas ${ }^{1}$
}

1 Universidad de las Américas, A. C.

\section{Correspondencia}

Escuela de Psicoterapia Combinada Compleja, email: tonghuitong@yahoo.com.mx. Privada de Aurora 21, Ciudad de México, CP 04000.

\section{Resumen}

Se construye el fundamento teórico necesario para la unificación de la psicoterapia a través de la conjunción de doce teorías pertenecientes a las Ciencias de la Complejidad. Específicamente se expone la manera en cómo se entretejen las teorías cognitiva, de la red epistemológica, de las nuevas lógicas, de la singularidad, de la bifurcación, de los atractores, de las catástrofes, de la fractalidad, del caos, del eclecticismo, de la transdisciplinaridad, de la diversidad, de la organización y de la complejidad. Al final se discute cómo todas ellas se expresan en el acto de la psicoterapia.

\begin{abstract}
The necessary theorical basement to unify the Psychotherapy is built though the joint of twelve theories which belong to the Complexity Sciences. It is shown the specific way of how are woven the following theories: cognitive, epistemological, the new logics, the singularity, bifurcation, atractors, catastrophes, chaos, eclecticism, transdisciplinarity, diversity, organization and complexity. How all of them are expressed in the act of psychotherapy is discussed.
\end{abstract}

Palabras clave: complejidad, transdisciplinaridad, caos, atractor, eclecticismo, singularidad, bifurcación, diversidad, organización.

La psicoterapia ha estado esperando muchos años para llegar a desarrollarse plenamente y así conformar un cuerpo unificado y combinado, sin divisiones internas, para poder alcanzar su máxima eficiencia y presentarse ante la comunidad de todas las otras disciplinas científicas para lograr así un 
status de mucho más respeto y reconocimiento de ellas y de la sociedad en general, además de transdisciplinarse con ellas de manera organizada. Esto no se logró en el pasado debido a que hacía falta que las bases epistemológicas de todos los modelos psicoterapéuticos encontraran un sustento común, lo cual ahora es viable a través de la articulación de la Teoría General de los Modelos y de las Catástrofes (Thom, 1987), la Teoría Fractal (Mandelbrot, 1989), la Teoría, de las Bifurcaciones (Koiter, 1976), la Teoría de los Atractores (Gleick, 2008), la Teoría de las Singularidades (Arnold, 1981), la Teoría del Caos, la Teoría Cognitiva, (Piaget, 1990), la Teoría de la Diversidad (Page, 2011), la Teoría de la Organización (Morín, 1999), la Teoría de las Nuevas Lógicas (McCown, Keiser \& Roden,1988; Olivas, 2001), la Teoría del Eclecticismo (Beitman y Yue, 2004; Beutler\& Harwood, 2000; Ferrater, 1969; Freeman, 1946; Norcross, 1986, 1987, 1990,1995; Robertson, 1995), la Teoría de la Red Epistemológica (Rescher, 1985, 1995, 1998), la Teoría de la Transdisciplinaridad (Klein, 1996; Morin, 2007; Nicolescu, 1994, 2002), y las Ciencias de la Complejidad (Nicolescu, 1994, 2002,2008; Morin, 1999, 2000,2004, 2007). Pero ¿Qué es lo que de ellas ha posibilitado la fundamentación de tal combinación y unificación? ¿Cómo se articulan? y ¿Qué características poseen? El objetivo de la presente investigación es contestar estas preguntas.

Gráficamente, la figura 1 muestra una idea aproximada de las relaciones del conjunto de todas estas teorías asumiendo que las interacciones, retroacciones y bucles entre ellas generan una circulación no causal sino Compleja entre todas (Compleja en el sentido de tal teoría). Se notará que en dicha figura la teoría cognitiva y la de la red epistemológica no aparecen ubicadas pero como se verá más adelante se encuentran relacionadas de una manera múltiple con las que sí aparecen.

Así, enseguida se expondrá el entramado en las disciplinas hasta aquí mencionadas.

1. Influencia de la Teoría de la Transdisciplinaridad (Klein, 1996; Morin, 2007; Nicolescu, 1994, 2002), a través de sus afluentes que son la meta, inter y poli disciplinaridad, propone que todas las disciplinas del conocimiento, y por extensión todos los modelos teóricos psicoterapéuticos con sus partes constituyentes, aquí colocados en lugar de las disciplinas, y por la acción de los psicoterapeutas, se interconecten conservando todas sus características individuales, es decir, se trans-conecten, lo cual significa que se derriben los muros teóricos y epistemológicos y se amplíen explorando sus propios límites actuales a través de combinaciones y recombinaciones. A su vez, la profundización en la investigación y en el progreso de los componentes de la psicoterapia debe cumplir con la tarea llamada metadisciplinarización (la investigación exhaustiva de una disciplina del conocimiento) que es una parte fundamental del avance del conocimiento transdisciplinario de acuerdo con autores como Klein (1996, 2001), Morín $(2004,2007)$ y Nicolescu $(2002$, 2008) y es una de las finalidades de cada modelo (Karasu, 1979). Como ejemplo de esta metadisciplinarización se podría citar el caso del modelo psicoanalítico freudiano, que con el paso del tiempo ha ido generando variaciones (Page, 2011) de sí mismo y ha llegado hasta el punto de fabricar modelos psicodinámicos de duración muy breve y de objetivos de tratamiento muy específicos, Esta situación también se muestra cuando el mismo modelo psicoanalítico va combinándose $y$ recombinándose de diferentes maneras con diversas partes de otros modelos diferentes, (Brooks-Harris, 2008) y de otros datos generados en diferentes disciplinas del conocimiento humano en general, realizando así una especie de búsqueda de sus conexiones. Un caso específico de lo mismo se ve en el llamado Modelo Analítico Cíclico de Wachtel (Wachtel, Kruck, \& Mckinney, 2005) en el que se articulan la teoría psicoanalítica con partes de los modelos conductual y sistémico.

En suma lo que la Teoría de la Transdisciplinaridad aporta es los fundamentos y motivos por los cuales se pueden reunir los modelos psicoterapéuticos, además de unir éstos al resto de los conocimientos científicos 


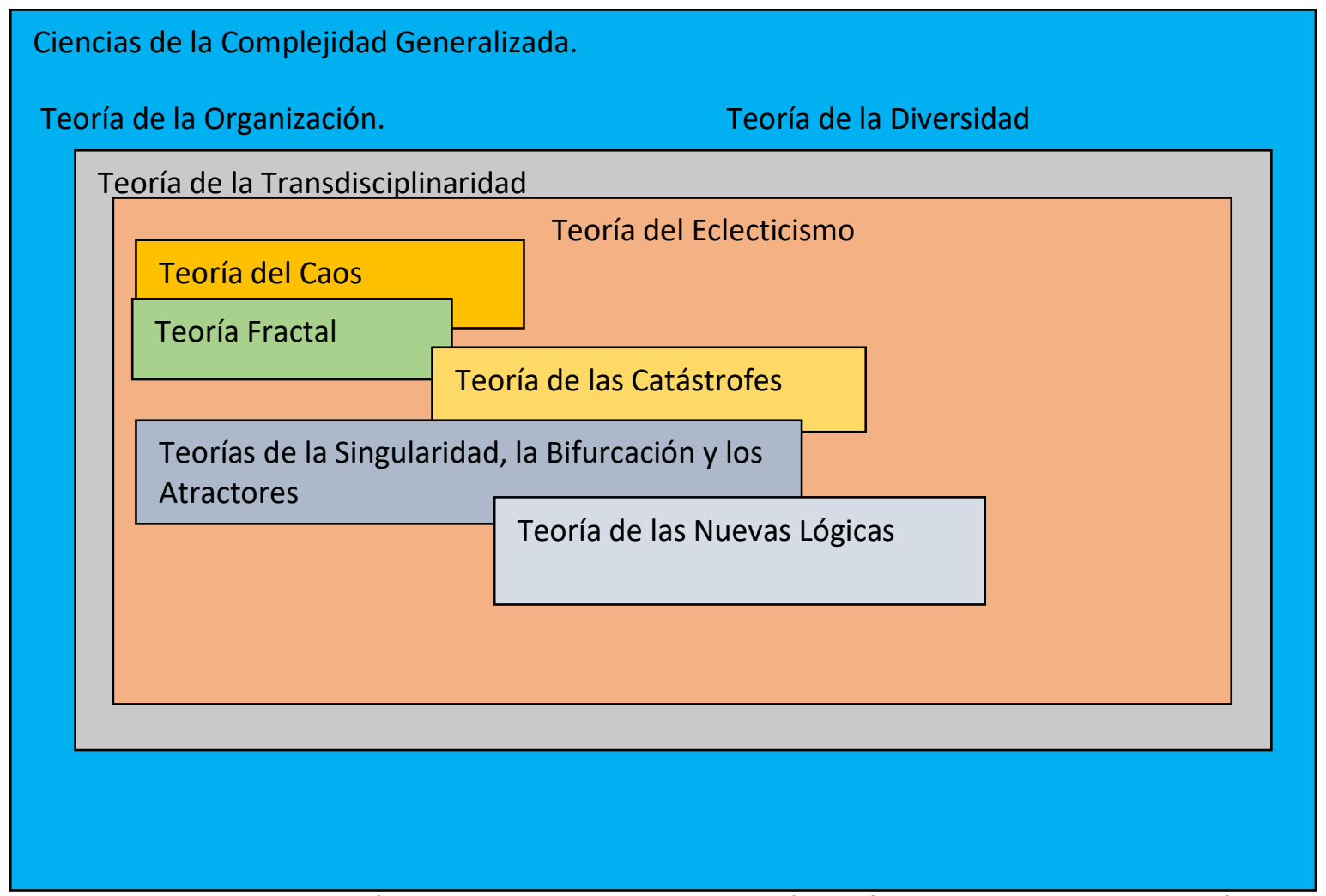

Figura 1. Las doce teorías que posibilitan la combinación unificadora de la Psicoterapia forman un conjunto en el que, como se observa, las más abarcativas son la de la Organización, la de la Diversidad y la de la Transdisciplinaridad.

2. Influencia de la Teoría de la Red Epistemológica (Rescher, 1985, 1995, 1998)

Esta Teoría plantea que las diferentes epistemologías pertenecen a una única red de epistemologías donde ninguna de ellas es superior a las otras y donde todas se complementan de manera organizada. Esta red posibilita que, diferentes epistemologías creadas con principios que se hayan planteado ya sea como diferentes o incluso como mutuamente excluyentes, formen un sistema incluyente a través de la teoría de la organización Compleja. La Red Epistemológica indicaría que en el caso de la psicoterapia, se podría constituir una red con las epistemologías que han dado origen a las diferentes teorías psicoterapéuticas lo cual obligará a diferentes modelos psicoterapéuticos, incluidas sus partes, a reunirse bajo los principios de tal organización, eliminando las guerras que se han presentado a todo lo largo de la psicoterapia. (Saltzman, \& Norcross, 1990).
En resumen, la Teoría de la Red Epistemológica aporta la posibilidad de reunir las epistemologías que fundamentan a cada modelo de psicoterapia en una red o Supersistema de epistemologías que serviría para reunirlas a todas sin algún criterio de exclusión aprovechando los conocimientos aportados por todos y sin que colisionen puesto que el acomodo adecuado de todas entre sí estaría dado por la organización derivada del resto de teorías que se ocupan específicamente de ésta como la Teoría del Caos (Campbell, 1993; Prigogine, 1999), La de las Catástrofes (Thom, 1987), y otras que abordaremos en este mismo documento.

Ahora bien, las dos teorías precedentes, la de la transdisciplinaridad y la de la red epistemológica, como se ve, pugnan por la armonización de las partes del conocimiento que tradicionalmente se han mantenido aisladas, dejando por un lado a las partes del conocimiento llamadas disciplinas y por otro a las llamadas epistemologías. Tal que si se aplicase esto 
a los modelos psicoterapéuticos y a sus partes ambas teorías facilitarían que éstos y estas se reuniesen armoniosamente.

Las perspectivas organizativas de los modelos de la psicoterapia puede llevar a ésta al campo más amplio de la metadisciplinaridad, la interdisciplinaridad, la polidisciplinaridad y la transdisciplinaridad, las cuales, se piensa, son los caminos para un verdadero avance en el conocimiento científico general, en el cual la psicoterapia está incluida (López, 2012; Klein, 1996 y 2001; Morín, 2004, 2007; y Nicolescu, 2002, 2008). ¿Hasta dónde se puede avanzar en la organización transdisciplinaria de los elementos de un sistema como el psicoterapéutico? La respuesta a esta pregunta está todavía por contestarse y tal vez como ocurre con el principio de incompletud en matemáticas según Gödel (Hofstadter, 2001) inevitablemente dentro de este Supersistema tal vez se encuentra un límite que no se pueda rebasar.

\section{Influencia de la Teoría General de los Modelos y de} las Catástrofes

Ahora bien, la desconexión de los modelos es inevitable debido a que, como lo expone la Teoría General de los Modelos (Thom, 1987), la manera en que las limitaciones cognitivas humanas eliminan el indeterminismo de los sistemas de fenómenos es creando modelos particulares, locales, parciales 0 especiales de fenómenos. Thom al dividir los modelos en formales (o deterministas) construidos para la predicción, y en modelos continuos (locales, cualitativos o particulares) establece que lo determinístico arriba inevitablemente a lo cualitativo. Al final, este matemático explica que los modelos formales deterministas finalmente llegan a formar, por efecto de infinidad de potenciales factores desconocidos e inobservables (los llamados atractores que son una especie de imanes), ciertas consecuencias extremadamente diversas que producen las denominadas singularidades (eventos únicos). catástrofes (saltos o cambios drásticos de las dimensiones de los eventos) en todo un sistema o en un subsistema Complejo (López, 2012). Esto implica que la creación de modelos separados en psicoterapia obedeció a ese mismo determinismo, es decir, se crearon modelos unitarios de psicoterapia para eliminar la Complejidad derivada de la enorme cantidad de fenómenos que se abordan en tal disciplina, de manera que, desde esta perspectiva, los modelos unitarios son un artificio de la Complejidad. Y siendo una división artificial la que engendró artificialmente la división de la psicoterapia en modelos, nada justifica no devolverle a ella su unidad.

En resumen, la Teoría de las Catástrofes o de los saltos drásticos provee los fundamentos para asumir que los diversos modelos de psicoterapia llegarían inevitablemente y sin anticiparlo a un punto de su desarrollo en el que ya no podrían avanzar individualmente y tendrían que "saltar" drásticamente hacia los otros modelos ya fuese por partes o con su todo, formando una especie de fenómeno de embudo que los conduce a un camino común.

\section{Influencia de la Teoría Fractal.}

La Teoría Fractal, del matemático Mandelbrot (1989), está relacionada con las iteraciones, conversiones y evoluciones de las "formas" (alternativas, sistemas, datos, eventos, modelos, dimensiones, etc.), atípicas, irregulares y "fracturadas", y que por ello se pueden medir en una gran variedad de maneras simultáneamente (Campbell, 1993). Un sistema fractal, (atípico o irregular) es lo que se tiene, por analogía, en un sistema de modelos psicoterapéuticos puesto que éste no tiene regularidad, uniformidad 0 estabilidad en sus características como sistema. Cuando se constata que las nubes no son esferas o redondas, las montañas no son conos perfectos, las costas no son curvas perfectas, las cortezas de los árboles no son lisas, los relámpagos no viajan en una línea recta (Mandelbrot, 1989), se podría también constatar que los sistemas de modelos psicoterapéuticos no son uniformes pues tienen variaciones, conversiones, evoluciones y transformaciones en todos sus aspectos, teóricos, estratégicos, técnicos, etc., y que además, a cada modelo de psicoterapia, se le podría 
evaluar 0 "medir" de maneras diferentes, simultáneamente, siguiendo la perspectiva fractal.

Además desde la perspectiva de la Teoría Fractal existen diversas maneras en las que las entidades de una colección se dividen o segmentan iterativamente (es decir, que desde cierto nivel de percepción se identifican como patrones autorreproducibles 0 iteraciones, que dan como resultado pautas 0 micropautas), como los tallos del vegetal llamado brócoli. En la psicoterapia esto es lo que en ocasiones se presenta en las sesiones de psicoterapia, pues en esta se generan combinaciones de micropatrones de interacciones entre el psicoterapeuta y su cliente y estos patrones se insertan dentro de otros patrones de interacción durante la sesión o conjunto de sesiones de psicoterapia. En psicoterapia algunos patrones de combinaciones de interacciones, psicoterapeuta-cliente, son claramente identificables mientras que otros permanecen menos notorios debido a lo complicado de su organización, ocurriendo lo mismo con las combinaciones de herramientas. Sin embargo en algunas ocasiones no existen patrones autorreproducibles y surgen combinaciones que se convierten en solitrones 0 eventos Complejos únicos o cuasi únicos, que tienen una probabilidad de repetición extremadamente pequeña e incluso nula (Peat, 1989). Un ejemplo de esto lo constituye el caso en el que un cliente agrediese físicamente a su psicoterapeuta.

En suma la Teoría Fractal provee los fundamentos para la comprensión de las iteraciones y de las combinaciones de iteraciones de patrones y micropatrones de las herramientas o de los aspectos teóricos de los modelos psicoterapéuticos, aclarando con ello los aparentes nudos gordianos de los eventos que ocurren en psicoterapia.

5. Influencia de la Teoría de las Bifurcaciones, Teoría de los Atractores y Teoría de las Singularidades

¿De una manera resumida a qué se refieren estas teorías?

1. Las Bifurcaciones son cambios cualitativos 0 topológicos que ocurren cuando aparecen pequeñas variaciones en el valor de algún fenómeno o entidad y traen consecuencias muy grandes en su derredor (Koiter, 1976).

2. Las Singularidades son puntos o circunstancias con características muy raras e impredecibles, apareciendo en la matemática como discontinuidades o funciones mal definidas que llevan al infinito o a valores extremos. (Arnold, 1981)

3. Los Atractores son eventos, fenómenos, entidades o fuerzas de cualquier tipo, imprecisas e imprevistas que "atraen" a otros fenómenos, entidades o fuerzas hacia cierta dirección o condición. (Gleick, 2008) Como se nota, estos tres elementos forman parte de las llamadas "catástrofes" que ocurren en todo y ayudan a entender cómo se generan cambios 0 "saltos" muy grandes, drásticos y espontáneos en los fenómenos, eventos o datos (Arnold, 1987; Thom, 1987; Woodcock y Davis, 1989) Ayudan a entender todo lo que se intersecta y se separa espontáneamente, describen las separaciones múltiples que ocurren en una colección de entidades, lo cual a su vez ayuda a comprender el fenómeno llamado cambio cuando éste no se puede explicar con otros métodos de observación o de medición. Este tipo de cambios raros pueden ayudar a explicar la dirección, la intensidad o la cualidad de las variaciones de atributos cuando ocurren la diversificación de los tipos y las diferencias en configuraciones o conexiones entre los métodos, técnicas, procedimientos, habilidades y/o competencias de los procesos psicoterapéuticos.

En suma, las Teorías de las Bifurcaciones, los Atractores y las Singularidades aportan explicaciones para entender la aparente rareza de las combinaciones de diferentes partes o de cuerpos totales de los modelos psicoterapéuticos.

6. Influencia de la Teoría del Caos

Primero que nada habrá que precisar que Briggs y Peat (1999), Chamberlain \& Bütz, (1988), Kellert (1994). y Prigogine, (1999) resumen la teoría del Caos, que es una teoría de la organización (Del orden, del no-orden y del desorden) en siete leyes: 1Ley del vórtice. 2-Ley de la influencia sutil, 3-Ley de la creatividad y la renovación colectivas. 4-Ley de lo simple y lo complejo. 5- Ley de los fractales y la razón, 
6- Ley de los rizos o bucles fractales de la duración, y 7- Ley de la corriente de una nueva percepción.

Para la psicoterapia. las aplicaciones de estas leyes se pueden describir de la manera siguiente: 1. Que existen características inobservables del tiempo implicado en ella; 2. Que emerge el cambio en los fenómenos característicos de ella; 3. Que aparece en ella algo que no se percibe con los métodos tradicionales de observación y medición; 4 . Que en ella sobreviene lo emergente o imprevisto; 5 . Que en ella aparece lo pequeño y lo muy grande; 6 . Que en ella ocurre lo razonable y lo no razonable; 7.Que es necesario abrir una nueva percepción para identificar y comprender cabalmente los sucesos que ocurren en ella.

En suma, la Teoría del Caos expone la organización existente en el fondo de fenómenos psicoterapéuticos aparentemente no ordenados y desordenados (así como lo hace con los fenómenos de otras disciplinas) contradiciendo la opinión de algunos autores como Norcross y Goldfried (2005a) quienes lamentan el "desorden" de la multiplicidad de los modelos psicoterapéuticos y de sus partes.

7.. Influencia de la Teoría de la No-linealidad

La Teoría de la No-linealidad (Campbell, 1993) reúne las características de la Teoría de la transdisciplinaridad, la Teoría del Caos, la Teoría Fractal, la Teoría de las Catástrofes, la Teoría de las Singularidades, la Teoría de las Bifurcaciones y la Teoría de los Atractores. La reunión de las características de todas estas teorías conforma una manera de abordar los fenómenos de las diferentes disciplinas científicas tal que sin ellas 0 con solamente algunas de ellas no se podían describir adecuadamente. Por ello todas estas teorías se han empezado a reconocer como Teorías No Lineales.

En suma las ciencias de la No Linealidad en su conjunto se convierten en una poderosa herramienta para enfocar las aristas más oscuras de la psicoterapia

7. Influencia de la Teoría de la Organización.
Si todo el conocimiento psicoterapéutico debe ser organizado ¿Cómo hacerlo? Siguiendo a Morín (1999) la organización es el concepto crucial que vincula la idea de interrelación a la idea de sistema, que une, transforma, produce y mantiene a este sistema; la Organización combina de forma diversificada los tipos de unión por dependencias fijas y rígidas, por interacciones recíprocas, por constituciones de elementos comunes a dos sistemas asociados que se convierten en subsistemas del sistema constituido, por retroacciones reguladoras, $y$, finalmente, por comunicaciones informativas. Así, la mejora se da transformando y formando (morfogénesis) porque: los elementos transformados en partes de un todo pierden cualidades y adquieren otras nuevas; la organización transforma una diversidad separada en una forma global; crea un contínuum donde había un discontinuo; forma un todo en el espacio y el tiempo y da paso a una realidad nueva que permanece en su forma, su existencia y su identidad. Con todo este esquema conceptual la acción organizativa prescribe cómo posicionar cada parte dentro del cuerpo total de la psicoterapia, es decir, tanto los elementos como las teorías y las epistemologías psicoterapéuticas existentes, y las prepara así para la transdisciplinarización, que ya se describió antes.

8. Influencia de la Teoría de la Diversidad.

Por su parte, la Teoría de la Diversidad, en consonancia con la Teoría de la Organización, que se acaba de describir, aborda de manera más detallada el tema de la combinación. Así, de acuerdo con la Teoría de la Diversidad de Page (2011), la diversidad se aplica a colecciones o conjuntos de entidades (fenómenos, eventos, objetos, ideas, sentimientos).

Según Page, la diversidad de una entidad puede significar cualquiera de tres características de una colección o conjunto: 1-Variación en algún atributo (lo largo de los picos de los pájaros pinzón), 2-Variación de tipos o clases (diferente mercancías de un supermercado), 3-Variación en configuración, es decir, en las conexiones o enlaces entre las partes de los tipos o clases (diferentes enlaces entre los átomos 
en una molécula).Es claro que, estos tres tipos de variaciones que producen diversidad en una colección o conjunto de entidades son el resultado de combinaciones (de atributos; de tipos o clases; y de diferentes configuraciones de alguna entidad). Algunas variaciones 0 diversificaciones son naturales, como ocurre con la diversidad de la que se habla en la teoría de la evolución de las especies de Darwin, y otras son intencionales, como ocurre con las variaciones que un músico ejecuta de una obra musical.

\section{Influencia de la Teoría Cognitiva.}

Las variaciones intencionales mencionadas en el apartado anterior, referente a la Teoría de la Diversidad, provienen de la acción cognitiva del observador que combina y subyace a la acción lógica de asociar, reunir, sumar, agrupar, clasificar, reunir, amalgamar, asociar, mezclar, integrar, unificar, componer, y religar (Brooks-Harris, 2008; Page, 2011; Piaget, 1990), y por lo tanto está siempre presente en todos los procesos cognitivos y meta cognitivos que se utilizan al percibir cualquier disciplina, incluyendo, obviamente, la psicoterapia.

En suma, la interacción de las teorías de la organización, de la diversidad y de la cognición, explica que los modelos y sus partes se combinen inevitablemente cada vez más.

10. Influencia del Eclecticismo Filosófico y Psicoterapéutico

El filósofo Diógenes Laercio propuso que inevitablemente existió una entidad de la cual todo lo demás se deriva mediante combinaciones, diversificaciones y variaciones. Esta postura ha sido aceptada por todas las disciplinas científicas de una u otra manera y diversos autores han generado incluso modelos psicoterapéuticos que explícitamente se nombran como eclécticos 0 integrativos, aceptando así la propuesta de Diógenes (Beitman y Yue, 2004; Beutler\& Harwood, 2000; Ferrater, 1969; Freeman, 1946; Norcross, 1986, 1987, 1990,1995; Robertson, 1995). Aunque esta postura de producción de nuevos conocimientos psicoterapéuticos dejo el ¿Cómo? abierto, sí puso las bases para las teorías que en adelante se expondrán aquí.

\section{Influencia de las Nuevas Lógicas}

¿Cuáles son las nuevas lógicas que influyen en la unión de los elementos de la psicoterapia? Habría que considerar las siguientes lógicas: la Fuzzy, la Intuicionista, la Monotónica, la Cuántica y las Paraconsistentes. Por ejemplo la lógica difusa que es una lógica que rechaza el principio del tercero excluido y propone un número infinito de valores de verdad, nos hace comprender los conjuntos matemáticos parciales o conjuntos lógicos fuzzy que proveen a la unificación de la psicoterapia con la explicación para entender que elementos parciales, imprecisos, difusos o no claros de algunos modelos de psicoterapia pueden entrar a formar parte de otros modelos o de partes de ellos, o que se puede construir todo un paquete de herramientas unidas imprecisamente, o que se puede hacer esto mismo solamente con partes pequeñas de modelos (McCown, Keiser \& Roden,1988; Olivas, 2001). En suma el análisis de los modelos psicoterapéuticos y de sus partes con la lupa de las nuevas lógica provee potenciales combinaciones de ellos/as.

12. Influencia de la Teoría de la Complejidad Generalizada.)

La Teoría de la Complejidad Generalizada (Morin, 2007) propone tejer, organizadamente, todo el conocimiento derivado del conjunto de todas las teorías antes expuestas, y como resultado de ello, resumidamente propone que en la psicoterapia existe lo siguiente: 1-Sensibilidad o influencia de las condiciones iniciales de la psicoterapia; 2Periodicidad-noperiodicidad, es decir repetición y norepetición de micro pautas psicoterapéuticas; , 3Escalamiento o recurrencia de las mismas pautas psicoterapéuticas en diferente tamaños; 4-Estados transicionales que se traducen como un estado de cambio del sistema psicoterapéutico por la aplicación del atractor que lo predispone a un cambio Caótico; 5-Atractores extraños, neutralizadores y repeledores en los eventos psicoterapéuticos; 6-Aspectos, eventos, fenómenos y lógica "difusos", "borrosos", "imprecisos" u "obscuros" (factores fuzzy) dentro de 
la psicoterapia (McCown, Keiser \& Roden,1988; Olivas, 2001); 7-Poli sistemicidad, es decir inclusión de múltiples sistemas en la psicoterapia; 8emergencia o espontaneidad de los eventos 0 fenómenos psicoterapéuticos; 9-Fractalidad 0 inclusión de los fenómenos fractales (véase arriba) en la psicoterapia; 10-Catastroficidad (véase arriba) dentro de la psicoterapia; 11-Bifurcabilidad (véase arriba) en la psicoterapia; 12- Avalanchas de fenómenos, fenómenos que reaccionan unidos y en cadena por acción de algún atractor, en la psicoterapia; 13-Desmoronamiento de fenómenos dentro de la psicoterapia, es decir, la dinámica de tales fenómenos consistente en que al reunirse de cierta forma cambian "en cascada", de manera impredecible como cuando se desmorona una pila de arena al sufrir alguna variación (Hofstadter, 2001); 14Aparición de sistemas de fenómenos psicoterapéuticos cuasi únicos o solitrones; y 15Transdisciplinaridad, es decir, inclusión de todos los eventos y fenómenos del Hipersistema humano. $\mathrm{Y}$, además de todo lo anterior, hay que recordar que a estos aspectos habría que agregar los eventos y fenómenos derivados de su interacción, retroacción y recursión sistémica.

\section{Discusión.}

En resumen, teóricamente ¿Cómo estas doce teorías determinan la combinación de los modelos psicoterapéuticos y de sus partes? De manera sintética se podría decir que los "saltos", las diversificaciones, las bifurcaciones, la influencia de "atractores", la ocurrencia de "catástrofes", la espontaneidad o fenómeno physis, los solitrones, las micropautas de la fractalidad de los fenómenos, y la organización, dentro del aparente no-orden de lo Caótico, en el sentido de la Teoría del Caos, se tejen, 0 articulan entre sí, aunque ello sea enormemente complicado. Así, estos fenómenos aparecen y se pueden detectar en lo que sucede en la interacción, entre el psicoterapeuta y su cliente, y ocurren sin que en general se les conceptúe de la manera que aquí se propone dentro de la psicoterapia. Ejemplos de estos fenómenos en la sesión de psicoterapia pueden ser por ejemplo los siguientes: los "saltos" podrían observarse en el cambio de temas de consulta imprevistos; los bucles o espirales de "recurrencia" en las emociones que aparecen en una sesión de psicoterapia; los cambios "drásticos" en las posturas del terapeuta y del cliente; la "espontaneidad" en los pensamientos espontáneos de ambos; la diversificación en las múltiples técnicas y 0 procedimientos que puede hacer el psicoterapeuta; las micropautas en el lenguaje o las preguntas dentro de la sesión; los eventos únicos de cualquier tipo en una cuasi única ocasión dentro del tratamiento; el aparente des-orden o Caos en la conversación entre el cliente y el psicoterapeuta.

Finalmente, a guisa de ejemplo y utilizando un lenguaje derivado de las diversas teorías discutidas hasta aquí se podría decir que se puede hacer que una herramienta "salte", es decir, pase, de un sistema psicoterapéutico (modelo) a otro; que una herramienta cambie "catastróficamente", es decir, enorme y cualitativamente rápido; que se puede producir una variación en una herramienta; que se pueden generar pautas o patrones diferentes a las/los que ya producen en un conjunto de herramientas; que se puede generar micropautas o micropatrones al potencializar las apariciones de pautas visibles 0 notorias con un conjunto de herramientas; que se puede generar intencionalmente una variación en una característica de una herramienta; que se puede generar intencionalmente una variación en una interacción o enlace de una parte de una herramienta con la de otra; que se puede generar una interacción o enlace entre diferentes clases de herramientas.

\section{Referencias}

Arnold, V. I. (1981). Singularity Theory. Selected papers. Cambridge: Cambridge University Press.

Arnold, V. I. (1987), Teoría de las Catástrofes. España. Alianza Editorial

Beitman, B. y Yue, D. (2004). Psicoterapia: programa de formación. N.Y.: Mason.

Beutler, L. E. \& Harwood, T. M. (2000). Prescriptive Psychotherapy: A practical guide to systematic treatment selection. New York: Oxford University Press.

Briggs, J. y Peat, F. D. (1999), Las siete leyes del caos. Barcelona: Revelaciones

Brooks-Harris, J. E. (2008) Integrative Multitheorical Psychotherapy. New York: Houghton Mifflin.

Campbell, A. B. (1993). Applied chaos theory. USA: Academic Press.

Chamberlain L. \& Bütz, M. R. Edit.(1988). Clinical Chaos: a therapist's guide to Nonlinear dynamics and therapeutic change. USA: Brunner-Mazel.

Ferrater, M. J. (1969). Diccionario de Filosofía. Argentina: Sudamericana. Barcelona: Paidós

Freeman, K. (1946). The pre-socratic philosophers. Great Britain: Basil Blackwell. 
Gleick, J. (2008) Chaos: Making a New Science. New York: Penguin Books.

Hofstadter, R. D. (2001). Gödel, Escher, Bach; un eterno y grácil bucle. México, Tusquets \& CONACYT.

Karasu, T. B. (1979). Toward unification of psychotherapies; a complementary model. The American Journal of Psychotherapy. 33: 555-563.

Kellert, S. H. (1994). In the wake of chaos; unpredictable order in dynamical systems. USA: The University of Chicago Press.

Klein, J. T. (1996). Crossing Boundaries; knowledge, disciplinarities and interdisciplinarities. London: University Press of Virginia.

Klein, J. T. (2001). Transdisciplinarity, joint problem solving among science, technology, and society; an effective way for managing complexity. Alemania: Birkhauser Verlag.

Koiter, W. T. (1976) "Current trends in the theory of buckling", Buckling of Structures. Proceedings of the IUTAM Symposium at Cambridge, pp. 1-16. Springer-Verlag, Berlín.

López, S. J. F. (2012). Las tendencias integrativas en psicoterapia. Tesis de Maestría, Universidad de las Américas, México.

Mandelbrot, B. B. (1989). Introduction to the fractal geometry of nature. New York. Rietman.

McCown, W., Keiser, R. \& Roden A. (1988). Cognitive psychology and chaos. En Chamberlain, L. \& Bütz, M. R. (Edit.) Clinical Chaos: a therapist's guide to Nonlinear dynamics and therapeutic change. USA: Brunner-Mazel.

Morin, E. (1999). El método; el conocimiento del conocimiento. Vol. III Madrid: Cátedra

Morin, E. (1999b). Los siete saberes necesarios para la educación del futuro. París: UNESCO.

Morin, E. (2000). Introducción al pensamiento complejo. España: Gedisa.

Morin, E. (2004). Sobre la interdisciplinaridad. Boletín \#2 del Centre International des Recherches et Etudes Transdisciplinaires. En Transdisciplina Creativa. Cecilia Suárez. Ed. 2002-2004. http://transdisciplina.tripoid.com

Morin, E. (2007). Complejidad restringida y complejidad generalizada o las complejidades de la Complejidad. Revista Internacional de Filosofía Iberoamericana y Teoría Social Venezuela: Universidad de Zulia. 38:107-119..

Nicolescu, B. (1994). La transdisciplinaridad; desvíos y extravíos. Turbulence \#1.
Nicolescu, B. (2002). Manifesto of Transdisciplinarity. USA: State University of New York Press.

Nicolescu, B. (2008). Transdisciplinarity; theory and practice. USA: Hampton Press.

Norcross, J. (1987). Casebook of eclectic psychotherapy. Ed., USA: Brunner/Mazel.

Norcross, J. (1986). Handbook of eclectic psychotherapy. USA: Brunner/Mazel.

Norcross, J. (1990). Commentary: Eclecticism misrepresented and integration misunderstood. Psychotherapy, 30: 402-403.

Olivas, V. (2001). La lógica borrosa y sus aplicaciones. Pag. Web arantxa.ii.uam.es/dcamacho/lógica/recursos/fuzzyinto-esp.pdf

Page, S. E. (2011). Diversity and complexity. USA: Princeton University Press.

Peat, D. F. (1989). Sincronicidad; puente entre mente y materia. Barcelona: Kairós.

Piaget, J. (1990). El nacimiento de la inteligencia. México. Grijalbo.

Prigogine, I. (1999), Las leyes del caos. Barcelona: Crítica

Rescher, N. (1985). Sistematización cognoscitiva. México: Siglo XXI.

Rescher, N. (1995). Lucha de los sistemas; un ensayo sobre los fundamentos e implicaciones de la diversidad filosófica. México: UNAM.

Robertson, M. H. (1995). Psychotherapy education and training. EUA: International Universities Press.

Rescher, N. (1998). Complexity. USA: Transaction Publisher.

Saltzman, N. \& Norcross, J. C. (1990). Therapy Wars; Contention and Convergence in Differing clinical Approaches. USA: Josey-Bass

Thom, R (1987). Estabilidad estructural $y$ morfogénesis; Ensayo de una teoría general de los modelos. España: Gedisa.

Wachtel, P. L., Kruck, J. C. \& Mckinney, M. K. (2005).Cyclical psychodynamics and integrative, relational Psychotherapy. En Norcross J. C. \& Goldfried, M. R. Edit. Handbook of Psychotherapy Integration. New York: Oxford University Press.

Woodcock, A. y Davis, M. (1989). Teoría de las catástrofes. España. Cátedra. 
